\title{
Análise da compreensão a respeito da terapia nutricional em Unidades de Terapia Intensiva de um hospital escola
}

\author{
Analysis of understanding about nutritional therapy in intensive care units of a teaching \\ hospital
}
Análisis de la comprensión de la terapia nutricional en unidades de cuidados intensivos de un hospital universitario

Emanuelle Negrão Quaresma ${ }^{1}$, Denise Bitar Vasconcelos Villacorta ${ }^{2 *}$, Rafael de Azevedo Silva ${ }^{2}$, Adriana de Oliveira Lameira Veríssimo ${ }^{1}$, Milena Coelho Fernandes Caldato ${ }^{3}$.

\section{RESUMO}

Objetivo: Conhecer as percepções dos médicos intensivistas de um hospital escola de Belém/PA sobre terapia nutricional. Métodos: Este foi um estudo transversal, descritivo e quantitativo realizado através de um questionário adaptado. A população-alvo foi composta de médicos atuantes nas Unidades de Terapia Intensiva de um hospital escola. O processo estatístico foi realizado nos softwares: SAM (Statistical Analysis Model) e BioEstat versão 5.3. Resultados: Avaliou-se 25 médicos plantonistas das UTI's em que 48\% possuem residência médica em terapia intensiva. Do total, $68 \%$ dos médicos responderam ser a perda de peso de $10 \%$ em um mês a considerada significativa para o início do suporte nutricional no paciente grave, $80 \%$ disse iniciar a nutrição em um paciente séptico na UTI em até $48 \mathrm{~h}$. Em suas práticas diárias, $75 \%$ dos intensivistas responderam que costumam decidir sobre o início da dieta enteral, porém $65 \%$ concorda em parte que possui dificuldade em reconhecer pacientes em risco nutricional em terapia intensiva e graduam-se com nota média de 5,5 a respeito de conhecimentos sobre suporte nutricional. Conclusão: Logo, os intensivistas conhecem pouco sobre terapia nutricional sendo necessária a criação e instituição de um protocolo em Terapia Nutricional (TN) a ser aplicado na UTI do hospital.

Palavras-chave Terapia Nutricional, Conhecimento, Unidades de Terapia Intensiva.

\begin{abstract}
Objective: To know the perceptions of intensive care physicians of a teaching hospital in Belém / PA about nutritional therapy. Methods: This was a cross-sectional, descriptive and quantitative study conducted through an adapted questionnaire. The target population consisted of doctors working in the intensive care units of a teaching hospital. The statistical process was performed using the software SAM (Statistical Analysis Model) and BioEstat version 5.3. Results: Twenty-five ICU physicians on duty were evaluated, $48 \%$ of whom had intensive care residency. Of the total, $68 \%$ of physicians reported that $10 \%$ weight loss in one month was considered significant for initiation of nutritional support in critically ill patients, $80 \%$ said they started nutrition in a septic ICU patient within 48 hours. In their daily practices, $75 \%$ of intensivists answered that they usually decide on the beginning of the enteral diet, but $65 \%$ agree in part that they have difficulty recognizing patients at nutritional risk in intensive care and graduate with an average grade of 5.5 to respect of knowledge about nutritional support. Conclusion: Therefore, intensivists know little about nutritional therapy and it is necessary to create and institute a protocol on Nutritional Therapy (TN) to be applied in the hospital ICU.
\end{abstract}

Key words: Nutrition Therapy, Knowledge, Intensive Care Units.

${ }^{1}$ Fundação Hospital de Clínicas Gaspar Viana (FHCGV), Belém/Pará. *E-mail: denibvv@gmail.com ${ }^{2}$ Centro Universitário Metropolitano da Amazônia (UNIFAMAZ), Belém/Pará.

${ }^{3}$ Universidade do Estado do Pará (UEPA), Belém/Pará. 


\section{RESUMEN}

Objetivo: Conocer las percepciones de los médicos de cuidados intensivos de un hospital universitario en Belém / PA sobre la terapia nutricional. Métodos: Este fue un estudio transversal, descriptivo y cuantitativo realizado a través de un cuestionario adaptado. La población objetivo consistía en médicos que trabajaban en las unidades de cuidados intensivos de un hospital universitario. El proceso estadístico se realizó utilizando el software SAM (Modelo de Análisis Estadístico) y BioEstat versión 5.3. Resultados: Veinticinco médicos de la $\mathrm{UCl}$ en servicio fueron evaluados, el $48 \%$ de los cuales tenían una residencia en cuidados intensivos. Del total, el $68 \%$ de los médicos informaron que la pérdida de peso del $10 \%$ en un mes se consideró significativa para el inicio del soporte nutricional en pacientes críticos, el $80 \%$ dijo que comenzó la nutrición en un paciente séptico de la $\mathrm{UCl}$ dentro de las 48 horas. En sus prácticas diarias, el $75 \%$ de los intensivistas respondieron que generalmente deciden sobre el comienzo de la dieta enteral, pero el $65 \%$ está de acuerdo en parte en que tienen dificultades para reconocer a los pacientes con riesgo nutricional en cuidados intensivos y se gradúan con una calificación promedio de 5.5 a respeto del conocimiento sobre soporte nutricional. Conclusión: Por lo tanto, los intensivistas saben poco acerca de la terapia nutricional y es necesario crear e instituir un protocolo sobre terapia nutricional (TN) para aplicar en la $\mathrm{UCl}$ del hospital.

Palabras clave: Terapia Nutricional, Conocimiento, Unidades de Cuidados Intensivos.

\section{INTRODUÇÃO}

Alimentar-se é uma necessidade humana básica, pois os nutrientes são fontes de energia e constituintes essenciais para o crescimento e sobrevivência dos seres vivos. Problemas na obtenção desses nutrientes podem resultar em condições patológicas (HERMANN AP e CRUZ EDA, 2008).

A desnutrição é uma desordem do estado nutricional, consequência de ingestão deficiente, alteração do metabolismo ou má nutrição. Sua resultante está relacionada a condições pré-mórbidas, tempo de ingestão insuficiente e concomitância de outras doenças. Pacientes desnutridos tem o seu tempo de internação aumentado, assim como sua morbimortalidade. Nos pacientes críticos observa-se um comprometimento da função imune e do drive ventilatório, fraqueza dos músculos respiratórios, elevando o número de dias em ventilação mecânica e, além disso, o aumento no risco de desenvolver úlceras de pressão; queda na absorção de nutrientes pelo intestino; aumento de infecções e da mortalidade, assim como aumento dos custos hospitalares. Na Unidade de Terapia Intensiva (UTI) a desnutrição acomete até $40 \%$ dos pacientes (HERMANN AP e CRUZ EDA, 2008; AMIB, 2013; SILVA RF, et al, 2014).

Apesar de este conceito estar muito bem estabelecido na literatura, persiste uma negligência com a atenção nutricional dada ao paciente dentro do hospital, principalmente na UTI, onde tudo parece prioritário em detrimento da terapia nutricional (AZEVEDO LCP, et al, 2015).

Durante décadas, na UTI, a administração de nutrientes era vista apenas como uma medida de suporte. Entretanto, pesquisas vêm mostrando que esse papel dentro da unidade pode ser muito maior e que, se realizado de maneira eficiente, pode resultar em redução de complicações (MUNOZ KFF, et al, 2016).

A terapia nutricional constitui-se em um elemento importante no cuidado ao paciente grave. $O$ estado nutricional é um dos maiores determinantes no curso desses pacientes, especialmente naqueles submetidos a prolongado estresse catabólico. Nestes, a desnutrição se correlaciona com mau prognóstico. Sendo assim, a relação entre o prognóstico do paciente crítico e a instituição de suporte nutricional é bem estabelecida (CUNHA HFR, et al, 2010; RIBEIRO PC, 2015).

No paciente grave, é de extrema importância que o suporte nutricional comece precocemente e esperase que ao passar de três dias o paciente esteja recebendo pelo menos dois terços de suas necessidades nutricionais, porém, estudos relatam que 40 a $60 \%$ dos pacientes aptos para o início de terapia nutricional ainda estão sem receber alimento dentro de 48h de admissão na UTI (AZEVEDO LCP, et al, 2015; MUNOZ KFF, et al, 2016). 
O adequado tratamento nutricional no paciente enfermo pode resultar numa evolução melhor da doença de base gerando maior sobrevida e menor custo hospitalar haja vista já ser confirmado a necessidade nutricional complexa (DIESTEL CF, et al, 2013).

Muitos são os fatores responsáveis pela falta de conhecimento no que diz respeito à nutrição e diagnóstico nutricional, entre eles destaca-se a pequena preocupação com o ensino, em nível de graduação e pósgraduação dos cursos da área da saúde, sobre o referido assunto (PAULO DA, et al, 2013).

A soma do pouco conhecimento sobre os benefícios nutricionais unidos a uma equipe de terapia intensiva sobrecarregada acaba resultando em negligência na qualidade do tratamento nutricional oferecido (PAULO DA, et al, 2013).

Partindo desse princípio, o presente trabalho teve como objetivo conhecer as percepções dos médicos intensivistas da Fundação Hospital de Clínicas Gaspar Vianna (FHCGV) (Belém - Pará) sobre terapia nutricional, assim como observar a necessidade de que sejam proporcionados treinamentos por parte da instituição apresentada, bem como chamar a atenção dos profissionais para a importância do tema em pauta.

\section{MÉTODOS}

Este foi um estudo transversal, descritivo e quantitativo realizado através de um questionário adaptado de um artigo publicado em 2010 (CUNHA HFR, et al, 2010). O questionário foi modificado com substituição ou retirada de alternativas pouco aplicáveis à dinâmica da instituição por outras que são mais comuns ao serviço. A população-alvo foi composta de profissionais médicos (plantonistas e rotina) atuantes nas Unidades de Terapia Intensiva da FHCGV pois o primeiro item da prescrição médica é a dieta sendo assim, a pesquisa buscou analisar quão os médicos estão preparados para essa prescrição. Foram excluídos médicos residentes, por atuarem sob supervisão de terceiros. O questionário foi entregue pessoalmente aos participantes na forma impressa, pela própria pesquisadora, que obteve o consentimento livre e esclarecido de cada um e orientou a respeito do escopo do estudo e do preenchimento das alternativas. Os impressos não foram identificados.

As características individuais analisadas foram idade (anos), gênero (masculino ou feminino), porte de título de especialista ou residência em Medicina Intensiva, tipo de cargo exercido na UTI (plantonista, diarista e/ou coordenação), tempo (anos) de formatura, tempo (anos) de atuação em Medicina Intensiva e perfil de paciente que mais comumente costuma assistir.

As análises avaliaram as condutas tomadas no setor comparando-as com a conduta mais correta baseada na literatura médica em Terapia Nutricional. Foram aplicados métodos estatísticos descritivos e inferenciais. As variáveis quantitativas foram apresentadas por medidas de tendência central e de variação e tiveram a distribuição avaliada pelo teste de Shapiro-Wilk. As variáveis qualitativas foram apresentadas por distribuições de frequências absolutas e relativas. Para avaliar as diferenças conforme os grupos GA (Grupo A) e GB (Grupo B) foi aplicado o teste do Qui-quadrado de independências. Para avaliar a diferença entre as variáveis quantitativas foi aplicado o teste de t- Student e a ANOVA (Análise de Variância). Para avaliar a tendência das variáveis qualitativas foi aplicado o teste do Qui-quadrado de aderência. Foi previamente fixado o nível de significância alfa $=0.05$ (margem de erro alfa de 5\%) para rejeição da hipótese de nulidade. $O$ processamento estatístico foi realizado nos softwares: SAM (Statistical Analysis Model) e BioEstat versão 5.3.

O estudo foi submetido e aprovado pelo Comitê de Ética em Pesquisa (CEP) sob CAAE: 73117817.0.0000.0016.

\section{RESULTADOS}

O presente estudo avaliou características do conhecimento e percepção em terapia nutricional de $n=25$ médicos plantonistas das UTIs da FHCGV. Para fins de análise os médicos foram divididos em dois grupos. No Grupo A (GA, com sexo masculino $57,1 \%$ ) ficaram os médicos que trabalham em terapia intensiva até 15 anos. O Grupo B (GB, com sexo masculino $54,5 \%$ ) foi composto pelos médicos que trabalham em terapia 
intensiva entre 15 e 25 anos. No Grupo A os médicos tem idade de $36 \pm 4$ anos. No Grupo B, os médicos têm idade de $48 \pm 3$ anos. Na amostra Geral ( $n=25$, sexo masculino $56 \%$ ) os médicos têm idade de $41 \pm 7$ anos. $A$ comparação GA x GB na Tabela 1, mostra que não existe real diferença entre os grupos ( $p>0.05)$.

Como mostrado na Tabela 1, do total de médicos entrevistados, $48 \%$ possuem residência em Terapia intensiva e 44\% tem título de especialista da AMIB (Associação de Medicina Intensiva Brasileira). A maioria referiu ocupar mais de $75 \%$ de sua carga horária semanal de trabalho com a terapia intensiva, sendo que $100 \%$ exercem função de plantonista, porém, entre eles há sete (7) com cargo de diarista e cinco (5) com cargo de chefia. O perfil de pacientes que mais costumam acompanhar são os clínicos críticos (92\%) (Tabela 1).

Tabela 1 - dados demográficos de $n=25$ médicos plantonistas das Unidades de Terapia Intensiva da Fundação Hospital e Clínicas Gaspar Vianna. Belém/PA, ano 2017.

\begin{tabular}{|c|c|c|c|c|c|c|c|c|}
\hline & \multicolumn{2}{|c|}{ GA } & \multicolumn{2}{|l|}{ GB } & \multicolumn{2}{|l|}{ Geral } & Tendência & \multirow{2}{*}{$\begin{array}{c}\text { GA x GB } \\
\text { p-valor }\end{array}$} \\
\hline & $\mathbf{n}$ & $\%$ & $\mathbf{N}$ & $\%$ & $\mathbf{n}$ & $\%$ & p-valor & \\
\hline Sexo & & & & & & & 0,6873 & 0,7826 \\
\hline Masculino & 8 & 57.1 & 6 & 54.5 & 14 & 56.0 & & \\
\hline Feminino & 6 & 42.9 & 5 & 45.5 & 11 & 44.0 & & \\
\hline Formação em T.l. & & & & & & & $0,0118^{*}$ & 0,3112 \\
\hline Pós-graduação em T.I. & 1 & 7.1 & 1 & 9.1 & 2 & 8.0 & & \\
\hline Residência em T.I. & 9 & 64.3 & 3 & 27.3 & 12 & 48.0 & & \\
\hline Especialista AMIB & 4 & 28.6 & 7 & 63.6 & 11 & 44.0 & & \\
\hline Ativ.Prática continu. T.I. & 4 & 28.6 & 4 & 36.4 & 8 & 32.0 & & \\
\hline Tempo/semana assist. T.I. & & & & & & & 0,0772 & 0,9353 \\
\hline Menos que $25 \%$ & 2 & 14.3 & 1 & 9.1 & 3 & 12.0 & & \\
\hline $25 \%$ a $50 \%$ & 2 & 14.3 & 1 & 9.1 & 3 & 12.0 & & \\
\hline $50 \%$ a $75 \%$ & 5 & 35.7 & 4 & 36.4 & 9 & 36.0 & & \\
\hline Mais que $75 \%$ & 5 & 35.7 & 5 & 45.5 & 10 & 40.0 & & \\
\hline Funções exerce em T.I. & & & & & & & $<0,0001^{*}$ & 0,6589 \\
\hline Plantonista & 14 & 100 & 11 & 100 & 25 & 100 & & \\
\hline Diarista/Rotina & 3 & 21.4 & 4 & 36.4 & 7 & 28.0 & & \\
\hline Chefia/Coordenação & 2 & 14.3 & 3 & 27.3 & 5 & 20.0 & & \\
\hline Pós-graduação em T.I & 1 & 7.1 & 0 & 0.0 & 1 & 4.0 & & \\
\hline Paciente típico & & & & & & & $<0,0001^{*}$ & 0,5304 \\
\hline Pós-operatório & 11 & 78.6 & 6 & 54.5 & 17 & 68.0 & & \\
\hline Pacientes clínicos críticos & 12 & 85.7 & 11 & 100 & 23 & 92.0 & & \\
\hline Coronariano/cardio-intensiva & 12 & 85.7 & 8 & 72.7 & 20 & 80.0 & & \\
\hline Pacientes neuro-críticos & 6 & 42.9 & 2 & 18.2 & 8 & 32.0 & & \\
\hline Unidade ventilatória & 0 & 0.0 & 1 & 9.1 & 1 & 4.0 & & \\
\hline Unidade Semi-intensiva & 1 & 7.1 & 0 & 0.0 & 1 & 4.0 & & \\
\hline Outros & 0 & 0.0 & 1 & 9.1 & 1 & 4.0 & & \\
\hline
\end{tabular}

Fonte: Quaresma EN, Villacorta DBV, Silva RA, et al., 2019. *Tendência: Qui-quadrado aplicado a categorias independentes. GAxGB: Qui-quadrado comparando os grupos.

Quanto às atitudes e percepções a respeito da terapia nutricional (Tabela 2), observa-se que não há diferença entre os grupos GA e GB. Quando interrogados quanto ao percentual de perda de peso em 30 dias considerado significativo na decisão para início de suporte nutricional em pacientes críticos, a maioria (68\%) respondeu $10 \%(p<0,05)^{*}$. Em relação ao tempo de espera aceitável antes de iniciar nutrição enteral em pacientes sépticos na UTI, $80 \%$ considerou ser esse tempo de até $48 \mathrm{~h}$.

Nas suas checagens diárias na UTI, $88 \%$ considerou checagem de cabeceira elevada e 100\% afirma que verifica o controle glicêmico do paciente. Outros itens como checagem do aporte calórico (64\%), do volume de dieta nas $24 \mathrm{~h}(64 \%)$ e uso de agentes procinéticos (76\%) também merecem destaque (Tabela 2). 
Tabela 2 - Atitudes e percepções de n=25 médicos plantonistas das Unidades de Terapia Intensiva da Fundação Hospital e Clínicas Gaspar Vianna. Belém/PA, ano 2017.

\begin{tabular}{|c|c|c|c|c|c|c|c|c|}
\hline & \multicolumn{2}{|l|}{ GA } & \multicolumn{2}{|l|}{ GB } & \multicolumn{2}{|c|}{ Geral } & \multirow{2}{*}{$\begin{array}{l}\text { Tendência } \\
\text { p-valor }\end{array}$} & \multirow{2}{*}{$\begin{array}{l}\text { GAxGB } \\
\text { p-valor }\end{array}$} \\
\hline & $\mathbf{n}$ & $\%$ & $\mathbf{n}$ & $\%$ & $\mathbf{n}$ & $\%$ & & \\
\hline Perda de peso \% sup. & & & & & & & & \\
\hline Nutricional & & & & & & & $<0,0001^{*}$ & 0,6988 \\
\hline Perder $5 \%$ & 2 & 14.3 & 2 & 18.2 & 4 & 16.0 & & \\
\hline Perder $10 \%$ & 10 & 71.4 & 7 & 63.6 & 17 & 68.0 & & \\
\hline Perder $15 \%$ & 1 & 7.1 & 1 & 9.1 & 2 & 8.0 & & \\
\hline Perder $20 \%$ & 0 & 0.0 & 1 & 9.1 & 1 & 4.0 & & \\
\hline Outros & 1 & 7.1 & 0 & 0.0 & 1 & 4.0 & & \\
\hline Tempo p/ N.E., sepse, no & & & & & & & & \\
\hline CTI & & & & & & & $0,0009^{*}$ & 0,9314 \\
\hline Até $24 \mathrm{~h}$ & 6 & 42.9 & 5 & 45.5 & 11 & 44.0 & & \\
\hline Até $48 \mathrm{~h}$ & 5 & 35.7 & 4 & 36.4 & 9 & 36.0 & & \\
\hline Até $72 \mathrm{~h}$ & 2 & 14.3 & 2 & 18.2 & 4 & 16.0 & & \\
\hline Até 5 dias & 1 & 7.1 & 0 & 0.0 & 1 & 4.0 & & \\
\hline Até 7 dias & 0 & 0.0 & 0 & 0.0 & 0 & 0.0 & & \\
\hline $\begin{array}{l}\text { Utiliza algorit., prot., } \\
\text { checagem }\end{array}$ & & & & & & & $<0,0001^{*}$ & 0,9787 \\
\hline $\begin{array}{l}\text { Checa elevação } \\
\text { cabeceira }\end{array}$ & 12 & 85.7 & 10 & 90.9 & 22 & 88.0 & & \\
\hline Checa aporte calórico & & & & & & & & \\
\hline $24 \mathrm{~h}$ & 8 & 57.1 & 8 & 72.7 & 16 & 64.0 & & \\
\hline Checa aporte protéico & & & & & & & & \\
\hline $24 \mathrm{~h}$ & 7 & 50.0 & 3 & 27.3 & 10 & 40.0 & & \\
\hline Checa volume de dieta & 9 & 64.3 & 7 & 63.6 & 16 & 64.0 & & \\
\hline Controle glicêmico & 14 & 100.0 & 11 & 100.0 & 25 & 100.0 & & \\
\hline $\begin{array}{l}\text { Inst. de sonda pós- } \\
\text { pilórica }\end{array}$ & 0 & 0.0 & 1 & 9.1 & 1 & 4.0 & & \\
\hline $\begin{array}{l}\text { Inst.de nut.parent. e } \\
\text { enteral }\end{array}$ & 2 & 14.3 & 2 & 18.2 & 4 & 16.0 & & \\
\hline Interrupção dieta p proc. & 5 & 35.7 & 4 & 36.4 & 9 & 36.0 & & \\
\hline Mede resíduo gástrico & 5 & 35.7 & 3 & 27.3 & 8 & 32.0 & & \\
\hline Uso agentes prócinéticos & 10 & 71.4 & 9 & 81.8 & 19 & 76.0 & & \\
\hline
\end{tabular}

Fonte: Quaresma EN, Villacorta DBV, Silva RA, et al., 2019.

*Tendência: Qui-quadrado aplicado a categorias independentes. GAxGB: Qui-quadrado comparando os grupos. 
Ao descrever a situação de um paciente clinicamente estável, afebril, extubado, recuperando-se de sepse pulmonar, com diarréia líquida ( $5 x$ em $24 \mathrm{~h}$ ), a maioria adota como conduta a diminuição da taxa de infusão da dieta $(63 \%)$ e início de uso de probióticos $(50 \%)$, com forte significância estatística quando comparado com a interrupção da dieta por $24 \mathrm{~h}$ praticada por $13 \%$ dos médicos interrogados (Gráfico 1).

Gráfico 1 - Condutas tomadas diante de paciente com diarréia líquida

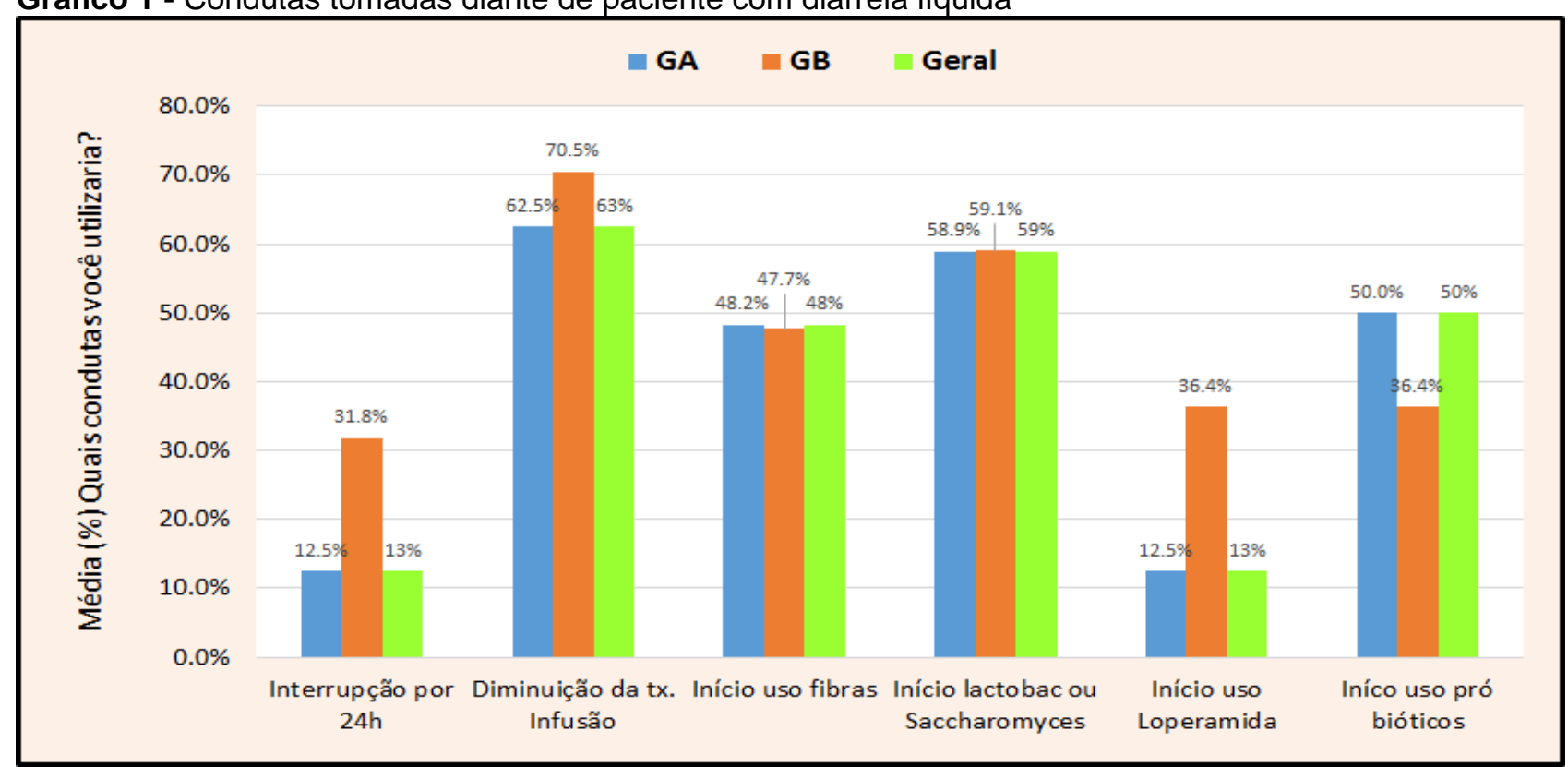

Fonte: Quaresma EN, Villacorta DBV, Silva RA, et al., 2019. Teste ANOVA, $p$-valor $=0,0003^{*}$, com pós-teste de Tukey.

Os intensivistas, em sua maioria $(75 \%)$, responderam que em suas práticas costumam decidir sobre 0 início de dieta enteral, com significância estatística ao se comparar com modificação do tipo de dieta a ser usada, onde apenas $35 \%$ decide sobre tal (Gráfico 2).

Gráfico 2 - Média de frequência que decide os seguintes elementos terapêuticos.

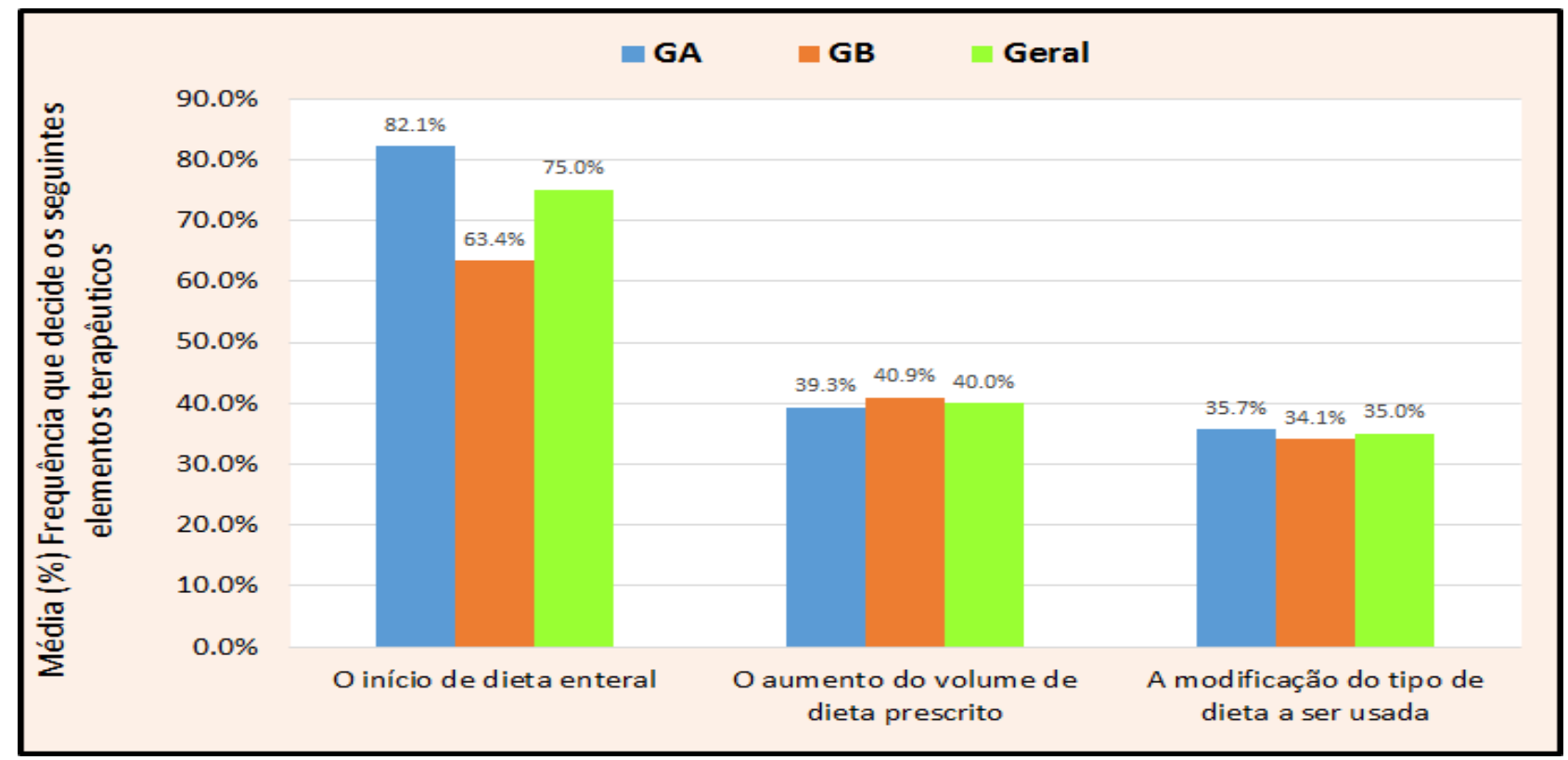

Fonte: Quaresma EN, Villacorta DBV, Silva RA, et al., 2019. Teste ANOVA, p-valor $=0,0002^{*}$, com pós-teste de Tukey. 
Sobre o volume $(\mathrm{ml})$ de resíduo gástrico considerado limítrofe na tomada de decisão sobre interrupção de dieta enteral não há um consenso: Não houve diferença entre os grupos $G A \times G B(p=0,7469)$ e a tendência é para a média $286,7 \pm 132,7 \mathrm{~mL}$, na faixa entre $155 \mathrm{ml}$ até $420 \mathrm{~mL}$.

Também não houve significância estatística para preferência de posicionamento de sonda para início de dieta enteral em pacientes com sepse pulmonar, sob Ventilação Mecânica e que não apresenta resíduo gástrico aumentado, onde $44 \%$ dos entrevistados considera colocação manual em nível gástrico. Quando interrogados sobre tempo após extubação para reiniciar dieta enteral, $28 \%$ consideram 5 horas, porém também não há significância neste resultado.

Um pouco mais da metade dos entrevistados (56\%) refere que concorda em parte com a seguinte afirmativa "Sinto dificuldade em reconhecer pacientes em risco nutricional em terapia intensiva" (Tabela 3). E a nota média com a qual graduaram seus conhecimentos sobre suporte nutricional foi 5,5 (Tabela 4).

Tabela 3 - Grau de concordância com a afirmativa: Sinto dificuldade em reconhecer pacientes em risco nutricional em terapia intensiva.

\begin{tabular}{|c|c|c|c|c|c|c|}
\hline & GA & & GB & & Geral & \\
\hline Grau de concordância & $\mathrm{n}$ & $\%$ & $\mathrm{n}$ & $\%$ & $\mathrm{n}$ & $\%$ \\
\hline Discordo plenamente & 2 & 14.3 & 0 & 0.0 & 2 & 8.0 \\
\hline Discordo em parte & 2 & 14.3 & 3 & 27.3 & 5 & 20.0 \\
\hline Neutro & 1 & 7.1 & 1 & 9.1 & 2 & 8.0 \\
\hline Concordo em parte & 7 & 50.0 & 7 & 63.6 & 14 & 56.0 \\
\hline Concordo plenamente & 2 & 14.3 & 0 & 0.0 & 2 & 8.0 \\
\hline
\end{tabular}

Fonte: Quaresma EN, Villacorta DBV, Silva RA, et al., 2019. Tendência: $p=0,0018^{*}$, GAxGB: $p$-valor $=0,4202$

Tabela 4. Graduação de conhecimento sobre suporte nutricional em terapia intensiva.

\begin{tabular}{llll}
\hline Grau de & Média dos grupos & \\
Conhecimento & GA & GB & Geral \\
\hline Média & 5.5 & 5.5 & 5.5 \\
Desvio padrão & 1.7 & 1.4 & 1.5 \\
Mediana & 6.0 & 6.0 & 6.0 \\
p-valor & GA x GB (p=0,8754) & $\mathrm{p}=0,0097^{\star}$
\end{tabular}

Fonte: Quaresma EN, Villacorta DBV, Silva RA, et al., 2019. *teste de Shapiro-Wilk

Fechando o questionário, os médicos intensivistas da FHCGV responderam com unanimidade que gostariam que o hospital oferecesse treinamento em Terapia Nutricional.

\section{DISCUSSÃO}

A Terapia Nutricional é parte integral dos cuidados do paciente crítico e envolve procedimentos terapêuticos com o objetivo de manutenção ou recuperação do estado nutricional por via oral, enteral ou parenteral. Na última década, várias pesquisas vêm apontando a existência e o agravamento de um déficit nutricional no ambiente hospitalar, com ingestão deficiente de macro e micronutrientes, acometendo principalmente os pacientes das UTI's (RIBEIRO PC, 2015; OLIVEIRA MM e SILVA MTG, 2016). 
Dessa forma, as organizações e sociedades profissionais criaram diretrizes para os cuidados nutricionais do paciente crítico a fim de subsidiar as decisões acerca da terapêutica (PASINATO VF, et al, 2013). A equipe de UTI (Unidade de Terapia Intensiva) é composta por diversos profissionais, dentre eles o nutricionista responsável possui o objetivo de avaliar as necessidades nutricionais dos pacientes e discutir o suporte nutricional com os médicos da UTI, que serão responsáveis por receitar a nutrição.

Procurou-se realizar neste estudo a avaliação de algumas práticas adotadas em terapia nutricional nas UTIs de um hospital escola e observou-se que $68 \%$ dos intensivistas consideram a perda de peso corporal de $10 \%$ em um mês como significativa para o início do suporte nutricional no paciente crítico. Porém, de acordo com a sociedade européia de nutrição, a perda de $5 \%$ do peso corporal em 1 mês já é suficiente para se preocupar em iniciar o suporte nutricional e deve ser levada em consideração (MCCLAVE AS, et al, 2016).

De acordo com as diretrizes clínicas européia, canadense, americana e, mais recentemente, a brasileira (DITEN), o uso da nutrição enteral para pacientes críticos hemodinamicamente estáveis deve ser considarado e de forma precoce, entre 24 e $48 \mathrm{~h}$ após a admissão na UTI, visto que nesse período há maior facilidade de tolerância à dieta, redução do risco de disfunção da barreira intestinal e infecções, reduzindo ainda o tempo de internação hospitalar e ventilação mecânica (CARTOLANO FDC, et al, 2009; CUNHA HFR, et al, 2010; SA PS, et al, 2013; AMIB, 2017; BRASPEN, 2019).

Na presente pesquisa $80 \%$ dos médicos plantonistas disseram iniciar dieta no paciente séptico em um tempo de até 48h, o que está de acordo com o preconizado na literatura. Em um estudo publicado em 2010, $84 \%$ dos participantes também afirmam iniciar a terapia nutricional de forma precoce (CUNHA HFR, et al, 2010).

Nos algoritmos de checagem em suas rotinas diárias na UTI, 100\% dos médicos responderam que observam o controle glicêmico, $88 \%$ checam elevação da cabeceira, $76 \%$ observam uso de procinéticos e $64 \%$ diz checar o aporte de calorias e volume de dieta infundido em $24 \mathrm{~h}$. O que chama atenção é o fato de apenas $40 \%$ fazer a checagem do aporte de proteínas nas $24 \mathrm{~h}$ e $16 \%$ instituir nutrição parenteral associada a enteral. Na pesquisa de Cunha e colaboradores, $42 \%$ checaram aporte de proteínas e $46 \%$ disseram introduzir nutrição parenteral associada à enteral quando necessário (CUNHA HFR, et al, 2010).

De acordo com a BRASPEN, pacientes graves com gastroparesia preexistente, diabetes, uso de vasopressores ou sedativos, apresentam risco de intolerância alimentar e, portanto, beneficiam-se de procinéticos, uma vez que aceleram o esvaziamento gástrico. Sabe-se ainda que aproximadamente $50 \%$ dos pacientes sob ventilação mecânica invasiva apresentam hipomotilidade antral e redução do esvaziamento gástrico (RIBEIRO PC, 2015; BRASPEN, 2019)

Não houve ênfase para a instituição de dieta parenteral associada à enteral, porém, sabe-se que se não for possível atingir as necessidades calóricas depois de 7 a 10 dias de nutrição enteral exclusiva, deve-se considerar a nutrição parenteral suplementar (CASAER MP, et al, 2014; COHEN J e SINGER P, 2016).

Cada paciente apresenta um desafio individual, e a terapia nutricional deve ser adaptada às evoluções ou complicações imprevisíveis. Na UTI o paciente está predisposto ao catabolismo que acompanha a mobilização de proteínas para que o organismo produza energia e se recupere de suas lesões. A não checagem do aporte de proteínas expõe o paciente a riscos nutricionais (POLL FA e STEFANELLO MD, 2014).

Quanto ao volume de resíduo gástrico, observou-se que não houve um consenso. A Sociedade Brasileira de Nutrição Enteral e Parenteral (BRASPEN) recomenda não iniciar Terapia de Nutrição Enteral se o resíduo gástrico for superior a $500 \mathrm{ml} \mathrm{em} 6 \mathrm{~h}$. A mensuração do resíduo gástrico ainda é importante para avaliação do trato gastrointestinal contudo perdeu a importância para detecção de intolerância do trato para alimentação enteral (COHEN J e SINGER P, 2016).

Para os entrevistados, o posicionamento da sonda para início de dieta enteral no paciente séptico sem resíduo gástrico aumentado é de preferência colocado manualmente em nível gástico ou pós pilórico duodenal por via manual. Na literatura não há vantagem da posição de sonda pós-pilórica em relação à gástrica para 
oferta de nutrientes ao paciente grave se não houver distensão, refluxo ou gastroparesia. O importante é não retardar o início da terapia nutricional enteral (SA PS, et al, 2013).

Sabe-se que a administração da Terapia de Nutrição Enteral é dificultada por inúmeros fatores diretamente relacionados com a UTI e que a interrupção da dieta pode ocasionar inúmeros prejuízos aos pacientes críticos, que é diretamente proporcional ao tempo de pausa. A extubação é uma dessas causas e no presente estudo a maioria dos entrevistados considerou pausa de 5 horas após extubação para reiniciar a dieta, o que pode ser considerada excessiva (ROCHA AJSC, et al, 2017).

A percepção de não interromper a dieta em vigência de diarreia também foi um achado do estudo no qual se baseou este trabalho, onde $63 \%$ dos intensivistas disseram reduzir a vazão da dieta, o que não foi observado em outro estudo, onde as condutas foram variáveis e não houve diferença significativa (LORDANI CRF, et al, 2014).

Do total de médicos que participou desta pesquisa, $50 \%$ afirmou iniciar probióticos em pacientes com diarreia líquida e volumosa, porém a literatura cita que existem poucos resultados benéficos comprovados por pesquisas por diversas dificuldades, dentre elas a grande diversidade de espécies probióticos (SA PS, et al, 2013).

O desafio para muitos profissionais que atuam na UTI continua sendo determinar as necessidades nutricionais que se ajustem a cada paciente. No presente trabalho, $75 \%$ dos médicos disseram que decidem o início da dieta enteral, enquanto apenas 35\% diz decidir a modificação do tipo de dieta a ser usada. Cunha e colaboradores mostraram em seu trabalho que $54,4 \%$ dos entrevistados decidem sobre a modificação do tipo de dieta a ser usada (CUNHA HFR, et al, 2010).

Quando interrogados, $56 \%$ dos médicos responderam que concordam em parte com a seguinte afirmativa "Sinto dificuldade em reconhecer pacientes em risco nutricional na terapia intensiva" e graduaram com média de 5,5 o seu grau de conhecimento sobre suporte nutricional em terapia intensiva. Isso abre reflexão para a necessidade de instituir um protocolo de avaliação e manejo nutricional nos pacientes críticos a ser aplicado na UTI da FHCGV. Para fechar o questionário, os médicos participantes do estudo responderam em unanimidade que gostariam que a FHCGV promovesse treinamentos em terapia nutricional.

\section{CONCLUSÃO}

Verificou-se que a equipe de médicos intensivistas da FHCGV é, em sua maior parte, especialista com formação na área em que atua e adota práticas de terapia nutricional, em sua maioria, concordantes com a literatura atual. Porém, é importante ressaltar a clareza com que afirmam sentirem alguma dificuldade para reconhecer pacientes em risco nutricional e isto poderia ser amenizado coma criação e instituição de um protocolo em terapia nutricional a ser aplicado na unidade, assim como a promoção de cursos de treinamento em terapia nutricional a ser ofertado aos médicos da UTI visando melhoria e qualidade do serviço.

\section{REFERÊNCIAS}

1. AMIB (ASSOCIAÇÃO MÉDICA INTENSIVA BRASILEIRA). LASI. SEPSE. 2013. Disponível em: https://www.amib.org.br/pagina-inicial/

2. AMIB (ASSOCIAÇÃO DE MEDICINA INTENSIVA BRASILEIRA); Curso de Terapia Nutricional e UTI, $3^{\text {a }}$ edição 2016/2017. Disponível em: https://www.amib.org.br/pagina-inicial/

3. AZEVEDO LCP, et al. Medicina intensiva: abordagem prática. 2a edição. Barueri: Manole, 2015. 1111p.

4. BRASPEN. SOCIEDADE BRASILEIRA DE NUTRIÇÃO PARENTERAL E ENTERAL. Disponível em: http://www.braspen.com.br/sem-categoria/terapia-nutricional-enteral-precoce/. Acesso em: 8 jul. 2019.

5. CASAER MP, et al. Nutrition in the Acute Phase of Critical Illness. N Englj Med 2014; 370: 1227-1236.

6. CARTOLANO FDC, et al. Terapia nutricional enteral: aplicação de indicadores de qualidade. Rev Bras Ter Intensiva. 2009; 21(4):376-383.

7. COHEN J, SINGER P. Como simplificar a nutrição na unidade de terapia intensiva? Rev Bras Ter Intensiva. 2016; 28(4):369-372. 
8. CUNHA HFR, et al. Atitudes e percepções em terapia nutricional entre médicos intensivistas:um inquérito via internet. Revista Brasileira de Terapia Intensiva, 2010; 22(1): 53-63.

9. DIESTEL CF, et al. Terapia nutricional no paciente crítico. Revista HUPE, 2013; 12(3): 78-84.

10. HERMANN AP, CRUZ EDA. Enfermagem em nutrição enteral: investigação do conhecimento e da prática assistencial em hospital de ensino. Cogitare Enfermagem, 2008; 13(4): 520-525.

11. LORDANI CRF, et al. Conhecimento de profissionais de unidade de terapia intensiva sobre diarréia. Rev Bras Ter Intensiva. 2014; 26(3):299-304.

12. MCCLAVE SA, et al. Guidelines for the Provision and Assessment of Nutrition Support Therapy in the Adult Critically III Patient: Society of Critical Care Medicine (SCCM) and American Society for Parenteral and Enteral Nutrition (A.S.P.E.N). JPEN; 2016; 40(2): 159-211.

13. MUÑOZ KFF, et al. Nutrologia e nutrição em unidade de terapia intensiva: sinergia em busca de excelência. International Journal of Nutrology, 2016; 9(1): 109-117.

14. OLIVEIRA MM, SILVA MTG. A importância da terapia nutricional nas Unidades de Terapia Intensiva. BRASPEN 2016; 31(4): 347-56.

15. PASINATO VF, et al. Terapia nutricional enteral em pacientes sépticos na unidade de terapia intensiva: adequação às diretrizes nutricionais para pacientes críticos. Rev Bras Ter Intensiva. 2013; 25(1):17-24.

16. PAULO DA, et al. Conhecimentos e atitudes de cirurgiões frente aos conceitos de terapia nutricional. Revista do Colégio Brasileiro de Cirurgiões, 2013; 40(5): 409-419.

17. POLL FA, STEFANELLO MD. Estado nutricional e dieta enteral prescrita e recebida por pacientes de uma Unidade de Terapia Intensiva. ABCS Health Scl. 2014; 39(2):71-76.

18. RIBEIRO PC. Nutrição. São Paulo: Editora Atheneu, 2015. 236p.

19. ROCHA AJSC, et al. Causas de interrupção de nutrição enteral em unidades de terapia intensiva. Revista de Pesquisa em Saúde, 2017; 18(1): 49-53.

20. SÁ PS, et al. Terapia nutricional no paciente crítico. Revista HUPE, Rio de Janeiro, 2013; 12(3):78-84.

21. SILVA RF, et al. Conhecimento dos profissionais de saúde sobre procedimentos e interações medicamentosas em terapia nutricional. Com. Ciências Saúde, 2014; 24(3): 231-238. 